\title{
Efflux pump genes of the resistance-nodulation-division family in Burkholderia cenocepacia genome Paola Guglierame ${ }^{\dagger 1}$, Maria Rosalia Pasca ${ }^{\dagger 1}$, Edda De Rossi ${ }^{1}$, Silvia Buroni ${ }^{1}$, Patrizio Arrigo ${ }^{2}$, Giulia Manina ${ }^{1}$ and Giovanna Riccardi*1
}

\begin{abstract}
Address: ${ }^{1}$ Dipartimento di Genetica e Microbiologia, Università degli Studi di Pavia, Via Ferrata 1, 27100 Pavia, Italy and ${ }^{2}$ Consiglio Nazionale delle Ricerche, Istituto per lo Studio delle Macromolecole, Via De Marini 6, 16149 Genova, Italy

Email: Paola Guglierame - paola.g@ipvgen.unipv.it; Maria Rosalia Pasca - m.pasca@ipvgen.unipv.it; Edda De Rossi - derossi@ipvgen.unipv.it; Silvia Buroni - buroni.s@ipvgen.unipv.it; Patrizio Arrigo - arrigo@ge.ismac.cnr.it; Giulia Manina - giuliamanina@ipvgen.unipv.it; Giovanna Riccardi* - riccardi@ipvgen.unipv.it

* Corresponding author †Equal contributors
\end{abstract}

Published: 20 July 2006

BMC Microbiology 2006, 6:66 doi:10.1 |86/147|-2180-6-66
Received: 10 April 2006

Accepted: 20 July 2006

This article is available from: http://www.biomedcentral.com/I47I-2/80/6/66

(C) 2006 Guglierame et al; licensee BioMed Central Ltd.

This is an Open Access article distributed under the terms of the Creative Commons Attribution License (http://creativecommons.org/licenses/by/2.0), which permits unrestricted use, distribution, and reproduction in any medium, provided the original work is properly cited.

\begin{abstract}
Background: Burkholderia cenocepacia is recognized as opportunistic pathogen that can cause lung infections in cystic fibrosis patients. A hallmark of B. cenocepacia infections is the inability to eradicate the organism because of multiple intrinsic antibiotic resistance. As ResistanceNodulation-Division (RND) efflux systems are responsible for much of the intrinsic multidrug resistance in Gram-negative bacteria, this study aims to identify RND genes in the B. cenocepacia genome and start to investigate their involvement into antimicrobial resistance.
\end{abstract}

Results: Genome analysis and homology searches revealed 14 open reading frames encoding putative drug efflux pumps belonging to RND family in B. cenocepacia $J 2315$ strain.

By reverse transcription (RT)-PCR analysis, it was found that orf3, orf9, orf $I$, and orfl 3 were expressed at detectable levels, while orf 10 appeared to be weakly expressed in B. cenocepacia. Futhermore, orf 3 was strongly induced by chloramphenicol. The orf 2 conferred resistance to fluoroquinolones, tetraphenylphosphonium, streptomycin, and ethidium bromide when cloned and expressed in Escherichia coli KAM3, a strain lacking the multidrug efflux pump AcrAB. The orf2overexpressing $E$. coli also accumulate low concentrations of ethidium bromide, which was restored to wild type level in the presence of CCCP, an energy uncoupler altering the energy of the drug efflux pump.

Conclusion: The 14 RND pumps gene we have identified in the genome of $B$. cenocepacia suggest that active efflux could be a major mechanism underlying antimicrobial resistance in this microorganism. We have characterized the ORF2 pump, one of these 14 potential RND efflux systems. Its overexpression in E. coli conferred resistance to several antibiotics and to ethidium bromide but it remains to be determined if this pump play a significant role in the antimicrobial intrinsic resistance of $B$. cenocepacia. The characterization of antibiotic efflux pumps in $B$. cenocepacia is an obligatory step prior to the design of specific, potent bacterial inhibitors for the improved control of infectious diseases. Consequently, the topic deserves to be further investigated and future studies will involve systematic investigation on the function and expression of each of the RND efflux pump homologs. 


\section{Background}

Bacteria have evolved a variety of strategies to resist antibiotics such as enzymatic inactivation, target alteration, efflux, and permeability changes. Antibiotic resistance can be intrinsic or acquired. Intrinsic resistance is intimately associated to the biology of the organism and usually involves the ability to resist a large number of different classes of antibiotics, while acquired resistance occurs when a bacterium that is sensitive to antibiotics develops resistance by mutation or by acquisition of new DNA. Bacterial intrinsic drug resistance was thought to be a passive mechanism, based on the absence of the drug target or on the lack of permeability of the bacteria to a given drug. However, it is becoming increasingly evident that the intrinsic resistance of many bacteria to antibiotics depends on the constitutive or inducible expression of active efflux systems [1,2]. A typical example is Pseudomonas aeruginosa, which was long thought to be poorly susceptible to a large range of antibiotics of different classes due to the low level of permeability of its outer membrane to drugs. However, disruption of the gene encoding the MexB pump dramatically increases the susceptibility of $P$. aeruginosa to beta-lactams, tetracyclines, fluoroquinolones, and chloramphenicol [3], indicating that the resistance was mediated by efflux.

In the prokaryotic kingdom there are five major families of efflux transporters: MF (major facilitator), MATE (multidrug and toxic efflux), RND (resistance-nodulation-division), SMR (small multidrug resistance), and ABC (ATP binding cassette). All these systems utilize the proton motive force as an energy source, apart from the ABC family, which utilizes ATP hydrolysis to drive the export of substrates [4].

RND transporters exist in all kingdoms of living organisms, but seem to be involved in drug resistance especially in Gram-negative bacteria [5]. The RND transporters function as a protein complex spanning from the cytoplasmic membrane to the outer membrane. The recent resolution of the three-dimensional structures of TolC and AcrB from Escherichia coli and MexA and OprM from P. aeruginosa gave rise to a better understanding of the efflux mechanism in Gram-negative bacteria [6-10].

The efflux pump systems of the RND family are organized as tripartite efflux pumps. The pump in E. coli and other gram-negative bacteria has three components: a transporter (efflux) protein in the inner membrane (e.g., AcrB), a periplasmic accessory protein (e.g., AcrA), and an outer membrane protein (OMP) channel (e.g., TolC) [11]. AcrB captures its substrates within either the phospholipid bilayer of the inner membrane of the bacterial cell envelope or the cytoplasm [12] and transports them into the external medium via TolC [13]. The cooperation between
AcrB and TolC is mediated by the periplasmic protein AcrA. The genetic organizations of the genes encoding these tripartite efflux systems are also similar among different species. Typically, the genes are organized as an operon: the regulator gene is located adjacent to the gene encoding the periplasmic accessory protein, which is located adjacent to the gene encoding the efflux pump protein, which is located next to that for the OMP. The membrane fusion protein and the pump protein are usually cotranscribed. For some systems and/or species, the $\mathrm{OMP}$ is not collocated with the other genes, e.g., E. coli acr $A B$ and tolC [14] and P. aeruginosa mexXY and oprM [15].

RND efflux systems are also found in bacteria that exhibit cell wall properties to P. aeruginosa [16]. The Burkholderia cepacia complex (Bcc) is noted for their ability to cause opportunistic infections in humans, particularly in patients with cystic fibrosis (CF) [17]. Bcc infections of CF lung can result in asymptomatic carriage, chronic infection or "cepacia syndrome", which is characterized by a rapid decline in lung function that can include invasive disease [18]. The Bcc contains at least nine closely related species $[19,20]$, of which Burkholderia cenocepacia (originally $B$. cepacia genomovar III) is the most prevalent and has been most commonly associated with epidemic spread and increased clinical virulence $[20,21]$.

A hallmark of Bcc infections is the inability to eradicate the organism because of high-level multiple intrinsic antibiotic resistance [22].

The multiple antibiotic resistance of Bcc has been attributed to reduced outer membrane permeability [23-25], production of modifying enzymes such as $\beta$-lactamases [26], and alteration of antibiotic targets [27]. Much less information is available on drug efflux systems, and only a few multi-drug efflux pumps of the MFS and MATE families have been described in Bcc species [28,29]. Screening for chloramphenicol resistance of a cosmid library constructed from a CF clinical isolate allowed the identification of the first RND efflux operon in B. cenocepacia [30]. Within this operon, genes encoding a periplasmic lipaselike protein (llpE), a periplasmic linker (ceoA), a cytoplasmic membrane component $(c e o B)$, and an outer membrane protein (opcM) were identified [31]. CeoA, CeoB, and OpcM have homologs in $P$. aeruginosa and other Gram-negative bacteria. A significant difference from other prokaryotic multidrug efflux systems, including all RND pumps described to date, is the inclusion of a gene encoding a lipase-like protein in the B. cenocepacia efflux operon [31].

Aim of this study was to identify RND genes in the B. cenocepacia genome and start to investigate their involvement 
into antimicrobial resistance. Fourteen homologues of RND efflux pump genes were identified by in silico homology searches. By RT-PCR it was found that some of them were expressed at detectable levels in not inducing condition, i.e. growth in LB medium, while others were detected in inducing conditions, i.e. growth in LB medium in the presence of an antibiotic. Further, orf 2 gene conferred a multidrug resistant phenotype, when over-expressed in Escherichia coli KAM3 strain. Moreover, an energy-dependent ethidium bromide efflux was observed in E. coli KAM3 cells harbouring orf2 gene.

\section{Results and discussion \\ Analysis of RND drug transporters}

The B. cenocepacia J2315 strain was isolated in 1989 in Edimburgh (UK) from the sputum of a cystic fibrosis patient [[32], in this reference identified as CF5610]. The genome sequence of this strain is available within the Sanger website [33]. To identify the B. cenocepacia drug efflux proteins belonging to RND, we first scanned the $B$. cenocepacia predicted proteins for the presence of the transporter family signature characterized by a number of strongly conserved amino acid residues. The sequences of the motifs are: motif A ( G x s x v T v $\mathrm{F}$ F x g t D x x x A q vqVqnkLqxAxpxLPxxVqxqgxxvxk), motif B (alvlsaVFlPmaffg GxtGxiyrqfsiTxvs A m alsvxvalt t PAlcA), motif C (xxxGkxlxeAxx xa axxRLRPlLMTs LafilGvlPlai at GxAGa), and motif D (S i N t T l fglvlai GLlvD D A l VvVE $\mathrm{N} v$ e Rv l a e) [34], where $\mathrm{x}$ indicate any amino acid, capital letters show amino acids most frequently observed in a single position in more than $70 \%$ of the transport proteins, and lowercase letters display amino acid occurring in more than $40 \%$ of RND. By this method, fourteen possible RND homologues have been identified (here named ORF1-ORF14). In Table 1, the position of the residues encompassing the four typical motifs is indicated; it is noteworthy that the relative distances among the specific motifs appear well conserved in all the identified proteins.

Members of the RND family have 12 TMS, with two large loops between TMS1 and TMS2, and TMS7 and TMS8, respectively [5]. This organization appears well conserved in all the B. cenocepacia hypothetical RND transporters, except for ORFs 11 and 14, which present 11 and 10 TMS, respectively (data not shown). In order to assess a putative function of the identified transporters, an integrative analysis of protein motif databases was carried out, by using the InterProScan program. The proteins ORF1-ORF10 show the ACRIFLAVINRP, a 9-element fingerprint that provides a signature for members of the acriflavin resistance protein family as AcrB of E. coli [35], while the proteins ORF11-ORF14 show a 6-8 element fingerprint (Table 1). All hypothetical RNDs contain two transmembrane functional domains located within TMS4 and
TMS1 1 that coincide with the transporter family signature motifs $\mathrm{C}$ and $\mathrm{D}$, respectively (Table 1 ). The crystal structure of multidrug efflux transporter AcrB of E. coli [10] reveals that this protein is characterized by the four subdomains PN1, PN2, PC1, and PC2. The structural motifs PN1 and PN2 comprise the polypeptide segment between TMS1 and TMS2, while PC1 and PC2 comprise the segment between TMS7 and TMS8. All the B. cenocepacia RND transporters (ORF1-ORF14) show the four subdomains; the region with subdomains PN1 and PN2 coincides always with the transporter family signature motif A (Table 1). The TolC docking domain is composed of two subdomains, DN and DC, and these subdomains are found in all hypothetical RND proteins except the ORF11 that present only the DC subdomain (Table 1). In order to obtain a more exhaustive analysis about the structural and conformational properties of the ORFs, the amino acid sequences were analysed by Phyre program and the data were reported in Table 1. The ORF1-14 were compared to the RND proteins of $E$. coli and $P$. aeruginos $a$ by using the BLASTP program. Particularly, ORF1, ORF2, and ORF4 show strong identity with E. coli AcrB (60\%, 64\%, 66\%, respectively) and with $P$. aeruginosa MexB (55\%, 63\%, 64\%, respectively). ORF5 and ORF8 show identity with $P$. aeruginosa MexD (50\%, 56\%, respectively), while ORF9 and ORF10 show identity with $P$. aeruginosa MexF (56\%, $64 \%$, respectively). ORF1 1 and ORF12 are homologues of metal efflux transporters belonging to the RND family of E. coli and P. aeruginosa (data not shown). Particularly, ORF11 is highly homologous to CzrA protein of $P$. aeruginosa, involved in cadmium and zinc resistance [36].

Analysis of the upstream and downstream regions of the RND coding genes confirmed the association with both an AcrA/MexA homolog and a TolC/OprM homolog in a variety of arrangements (Figure 1). Indeed, orf1, orf2, orf3, orf4, orf8, orf9, orf10 (ceoB), orf11, orf12, orf13, and orf14 seem to be part of an operon that also included a mexA and an oprM homologs, thus containing all three of the components of the tripartite pump complex. Differently, orf5 appears to have only the membrane component, while orf6 and orf7 are apparently co-transcribed and in operon with a periplasmic membrane fusion protein (Figure 1). In $P$. aeruginosa, the genes encoding the RND transporter (MexB homolog) and periplasmic membrane fusion protein (MexA homolog) are always present, while the gene encoding the OM channel (OprM homolog) is not always present [37]. In E. coli, the genes for transporter and membrane fusion proteins (acrAB) occur together as an operon and the gene for the TolC occurs elsewhere on the genome [37]. In B. cenopacia the genetic organization of the RND orfs seems to resemble in part that of $P$. aeruginosa and in part that of E. coli. As AcrD pump of E. coli, orf5 seem to be transcribed alone in the genome of $B$. cenocepacia [38], while orf6 and orf7 are organized as the 
Table I: Predicted functional motifs for ORFI-ORFI4 of B. cenocepacia

RND CONSERVED

MOTIFS

FUNCTIONAL MOTIFS

STRUCTURAL

Motif s $A^{b}, B^{b}, C^{b}, D^{b} \quad$ Acriflavin resistance protein AcrB transmemdomains (ACRIFLAVINRP)c

brane domainc

BCAS0592 ORFI

Chromosome 3

BCAS0765 ORF2

Chromosome 3

BCALI675 ORF3

Chromosome I

BCAL282I ORF4

Chromosome I

BCALI778 ORF5

Chromosome I

BCALI079 ORF6

Chromosome I

BCALI080 ORF 7 Chromosome I
$38-134,445-493,826-$

1051, 272-503

$8-32,36-54,336-359,363-384,391-$

$415,445-468,470-493,552-569$

620-634

$38-134,445-493,809$ 1035, 272-503

$8-32,36-54,336-359,363-384,391-$ $415,445-468,470-493,553-570$,

$622-636$

38-133, 444-492, 8041027, 27I-502

8-32, 36-54, 335-358, 362-383, 3904I4, 444-467, 469-492, 55।-568, $618-632$

38-134, 445-493, 809$1035,272-503$

$8-32,36-54,336-359,363-384,39$ $415,445-468,470-493,553-570$, 622-636

40-136, 446-494, 8121037, 274-504

|0-34, 38-56, 338-36|, 365-386, $393-417$, 44

$40-136,438-486,792-$ $1019,266-496$

10-34, 38-56, 330-353, 357-378, $385-409,438-461,463-486,543-560$. 610-624
$40-134,438-486,853-$ 1084, 266-496
10-34, 38-56, 330-353, 357-378 $385-409,438-461,463-486,544-561$, $607-621$
$272-503,826-105 \mid$

272-503, 809-1035

27I-502, 804-1027

272-503, 809-1035

38-134, 566-672

274-504, 812-1037

$40-136,575-676$

266-496, 792-1019

40-136, 556-656

$266-496,853-1084$
AcrB pore domain

(PNI, PN2, PCI,

PC2 subdomains)

\section{AcrB/TolC dock- \\ ing domain (DN,}

DC subdomains)c

$38-134,565-669$

|82-27|, 720-825

-134, 566-672

$82-271,723-808$

$38-133,564-668$

$719-803,|8|-270$

|82-27|, 723-808

|84-273, 727-81 | transporter TolC docking domain

|89-265, 707-79|

Familyd

Multidrug efflux transporter AcrB pore

$$
\text { domain }
$$

Multidrug efflux transporter AcrB transmembrane domain transporter AcrB transmembrane domain

Multidrug efflux
transporter AcrB
transmembrane domain

Multidrug efflux transporter AcrB pore domain

|83-265, 704-776

$40-134,557-653$

265, 704-776
Multidrug efflux
Multidrug efflux transporter AcrB pore domain 


\begin{tabular}{|c|c|c|c|c|c|c|}
\hline $\begin{array}{l}\text { BCAM0926 ORF8 } \\
\text { Chromosome } 2\end{array}$ & $\begin{array}{c}38-134,445-493,803- \\
1027,272-503\end{array}$ & $\begin{array}{l}8-32,36-54,336-359,363-384,391- \\
415,445-468,470-493,552-569, \\
619-633\end{array}$ & $272-503,803-1027$ & $38-134,565-667$ & $|82-27|, 7 \mid 8-802$ & $\begin{array}{l}\text { Multidrug efflux } \\
\text { transporter AcrB pore } \\
\text { domain }\end{array}$ \\
\hline $\begin{array}{l}\text { BCAMI946 ORF9 } \\
\text { Chromosome } 2\end{array}$ & $\begin{array}{c}40-136,447-495,814- \\
1041,275-505\end{array}$ & $\begin{array}{l}10-34,38-56,339-362,366-387, \\
394-418,447-470,472-495,562-579 \\
631-645\end{array}$ & $275-505,8|4-104|$ & $40-136,575-678$ & $|85-274,729-8| 3$ & $\begin{array}{c}\text { Multidrug efflux } \\
\text { transporter TolC docking } \\
\text { domain }\end{array}$ \\
\hline $\begin{array}{l}\text { BCAM2550 ORF I0 } \\
\text { Chromosome } 2\end{array}$ & $\begin{array}{c}40-136,447-495,817- \\
1043,275-505\end{array}$ & $\begin{array}{l}10-34,38-56,339-362,366-387, \\
394-418,447-470,472-495,562-579 \\
631-645\end{array}$ & $275-505,817-1043$ & $40-136,575-68 \mid$ & $185-274,732-816$ & $\begin{array}{l}\text { Multidrug efflux } \\
\text { transporter AcrB pore } \\
\text { domain }\end{array}$ \\
\hline $\begin{array}{l}\text { BCAM07I3 ORF I I } \\
\text { Chromosome } 2\end{array}$ & $\begin{array}{c}42-135,480-503,822- \\
1049,167-513\end{array}$ & $\begin{array}{l}12-36,40-58,339-362,366-387 \\
480-503,553-570\end{array}$ & $\mid 67-513,822-1049$ & $42-135,566-673$ & $726-821$ & $\begin{array}{c}\text { Multidrug efflux } \\
\text { transporter TolC docking } \\
\text { domain }\end{array}$ \\
\hline $\begin{array}{l}\text { BCAM0435 ORF I } 2 \\
\text { Chromosome } 2\end{array}$ & $\begin{array}{c}42-135,454-502,807- \\
1043,269-511\end{array}$ & $\begin{array}{l}\mid 2-36,40-58,334-357,361-382 \\
389-413,552-569\end{array}$ & $269-511,807-1043$ & $42-135,565-610$ & $185-268,722-806$ & $\begin{array}{l}\text { Multidrug efflux } \\
\text { transporter AcrB pore } \\
\text { domain }\end{array}$ \\
\hline $\begin{array}{l}\text { BCALI8II ORF I } 3 \\
\text { Chromosome I }\end{array}$ & $\begin{array}{c}49-146,446-494,805- \\
1029,274-504\end{array}$ & $\begin{array}{l}47-65,339-362,393-4 \mid 7,446-469 \\
47|-494,554-57|\end{array}$ & $274-504,805-1029$ & $49-146,567-677$ & $|92-273,72|-804$ & $\begin{array}{l}\text { Multidrug efflux } \\
\text { transporter AcrB pore } \\
\text { domain }\end{array}$ \\
\hline $\begin{array}{l}\text { BCAS0582 ORF I } 4 \\
\text { Chromosome } 3\end{array}$ & $\begin{array}{c}40-136,438-486,832- \\
1060,266-496\end{array}$ & $\begin{array}{l}10-34,38-56,330-353,357-378 \\
385-409,438-46|, 463-486,55|-568\end{array}$ & $266-496,832-1060$ & $40-136,564-675$ & $|83-265,727-83|$ & $\begin{array}{l}\text { Multidrug efflux } \\
\text { transporter AcrB pore } \\
\text { domain }\end{array}$ \\
\hline
\end{tabular}




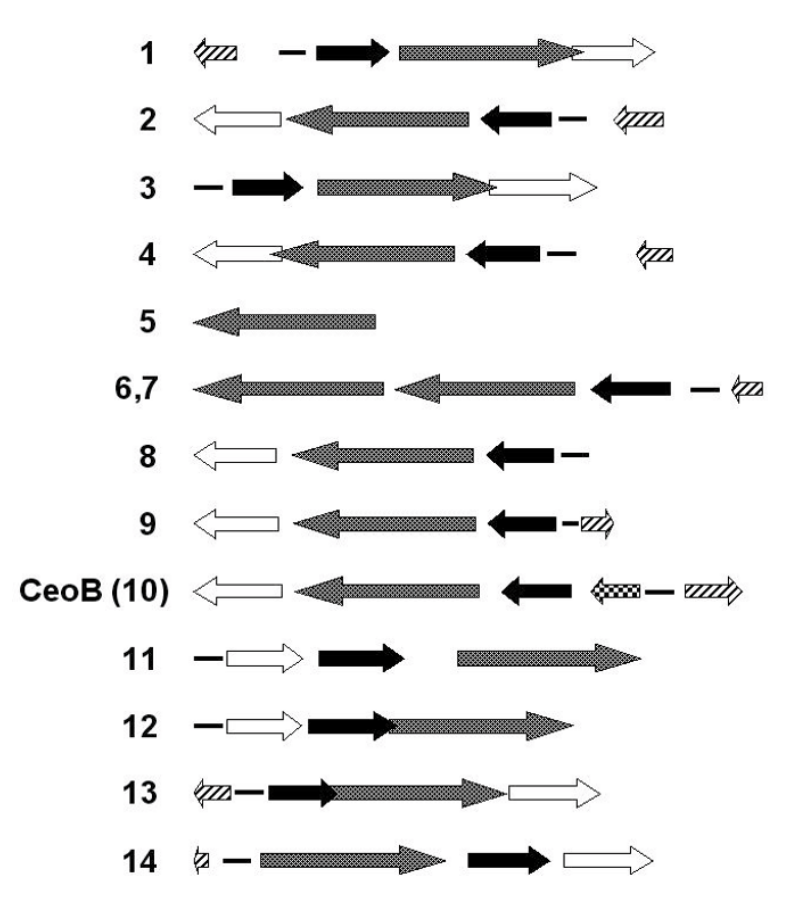

\section{Figure I}

Proposed RND efflux pump encoded by $B$. cenocepacia genome. The organization of the RND efflux pump genes identified in $B$. cenocepacia genome was achieved by bioinformatic tools as described in Methods. RND transporter-encoding genes are depicted as grey arrows (orflorfl 4), outer-membrane factor-encoding genes as white arrows, periplasmic membrane fusion protein-encoding genes as black arrows, and regulatory genes as arrows with stripes. Black lines indicate the regions of Stress-Induced DNA duplex Destabilization that could be associated with promoters. Genes encoding protein components of CeoABOpcM (6) are indicated as white arrow (opcM), grey arrow $(c e o B)$, black arrow (ceoA); arrows with stripes and squares indicated the ceoR and IIpE genes encoding a regulator and a putative periplasmic lipase-like protein, respectively.

genes PA2526-2527-2528 of P. aeruginosa that contains an additional RND transporter [39]. Many pump component-encoding operons contain a physically linked regulatory gene coding for either a repressor or an activator [40]. For example, expression of the mex $A B$-oprM operon is regulated by the product of the upstream and divergently transcribed mexR gene. Not surprisingly, clinical isolates that overexpress the MexAB-OprM system often carry mutations in the mexR gene [41]. In the case of orf1, orf2, $\operatorname{orf} 4, \operatorname{orf} 6-7, \operatorname{orf} 9, \operatorname{orf} 10, \operatorname{orf} 13$, and $\operatorname{orf} 14$ a hypothetical regulatory gene has been identified, divergently transcribed for all genes, except for the hypothetical operon containing the orf 2 , orf 4 , and orf $6-7$, respectively (Figure 1 ). The WEBSIDD program was used to analyse sequences encompassing the coding and intergenic regions of the hypothetical RND operons for the presence of SIDD (Stress Induced Duplex Destabilization) sites. These sites have a specific and statistically highly significant pattern of association with transcriptional regions, specifically with promoters because strand separation is a necessary step in the initiation of transcription [42]. All the ORFs present SIDD sites localized upstream of the first gene of the entire operon, except for the orf5.

\section{RT-PCR analysis of the B. cenocepacia genes coding for the RND transporters}

We found 14 genes codifying hypothetical RND drug transporters into B. cenocepacia genome. By RT-PCR analysis, we studied the expression of all these genes at the exponential phase of cells grown in LB medium. A clear expression of orf3, orf9, orf11, and orf13 was observed (Figure $2 \mathrm{~A}$, lanes $1,4,8,10)$, while orf10 appeared to be weakly expressed (Figure 2A, lane 6). The expression of the other orfs $(\operatorname{orf} 1, \operatorname{orf} 2, \operatorname{orf} 4, \operatorname{orf} 5, \operatorname{orf} 6-7, \operatorname{orf} 8, \operatorname{orf} 12$, and orf14) was not detectable, at least in our conditions (data not shown).

It has been demonstrated that orf10 corresponds to the previously described $c e o B$ gene belonging to ceo operon, which has been shown to be strongly induced by salicylate and chloramphenicol [31]. We decide to evaluated the expression of the 14 genes in $B$. cenocepacia cells grown in the presence of chloramphenicol (inducing conditions). In B. cenocepacia J2315 strain, the basal level of expression of the orf3 (Figure 2A, lane 1) was strongly increased by chloramphenicol (Figure 2A, lane 2). No induction could be detected in the case of orf $1, \operatorname{orf} 2, \operatorname{orf} 4, \operatorname{orf} 5, \operatorname{orf} 6-7, \operatorname{orf} 8$, orf12, and orf14 (data not shown) and no increased expression could be observed in the case of orf9, orf10, orf11, and orf13 (data not shown). However, the B. cenocepacia rRNA 16S expression was not affected by different growth conditions (Figure 2B, lanes 1 and 2), thus demonstrating that the differences detected in the amount of orf3 mRNA, under different growth conditions, are genuine.

As the ceo operon has been discovered as responsible for the MDR phenotype of a clinical strain [31], we can hypothesize that this RND efflux system is expressed as a result of a mutation. In our conditions, no transcriptional induction of orf10 could be detected also in the presence of salicylate (data not shown).

ORF3 showed $71 \%$ identity with $P$. aeruginosa MexY, an inducible efflux system that contributes to the natural resistance of $P$. aeruginosa to antibiotics [43]. Experiments involving real-time PCR in $P$. aeruginosa PAO1 showed induction of gene mexY by chloramphenicol, tetracycline, macrolides, and aminoglycosides [44]. To date, no infor- 

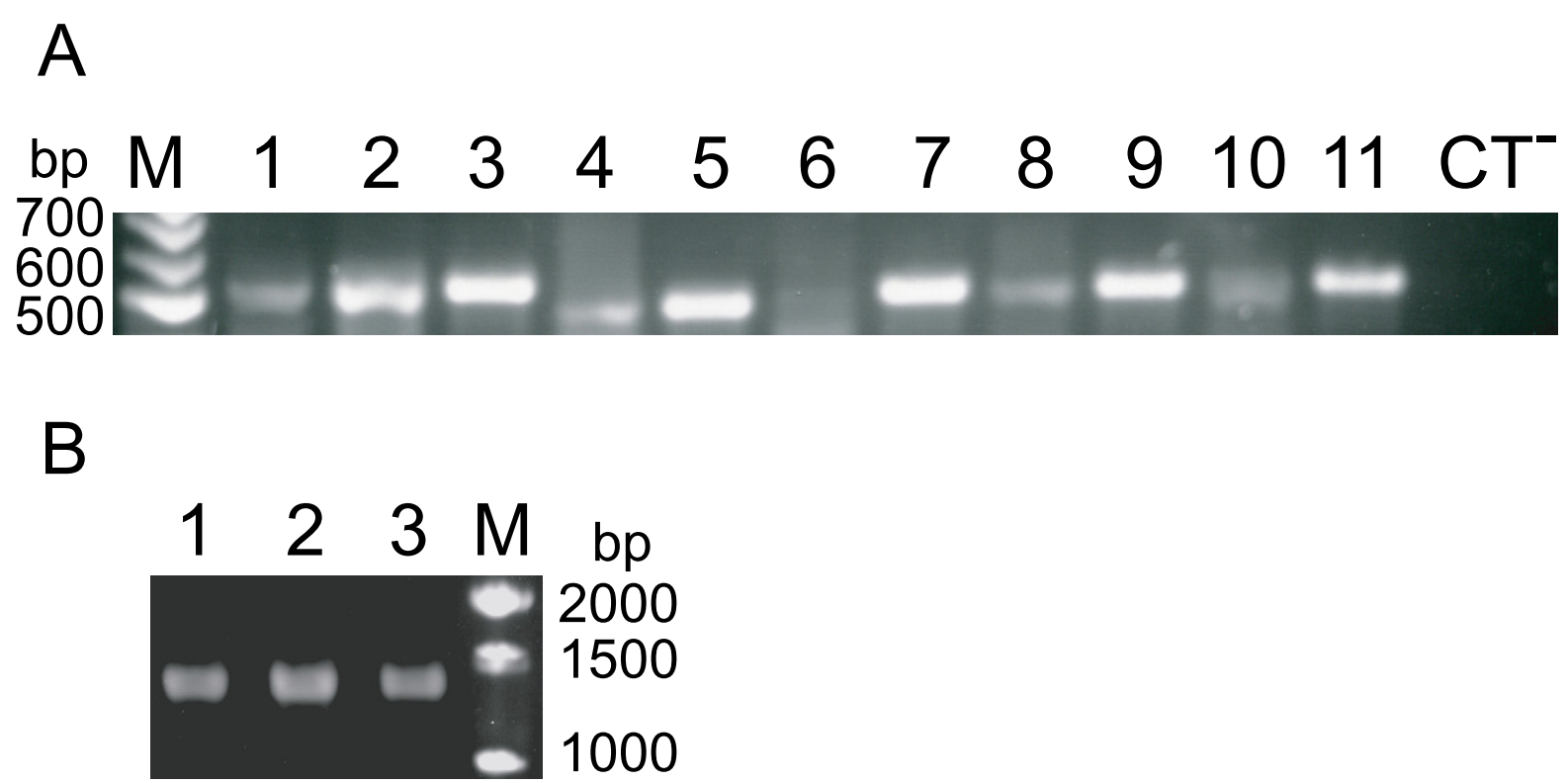

Figure 2

Expression of genes coding for the RND proteins from B. cenocepacia. Expression of genes coding for the RND proteins was evaluated by RT-PCR of RNA isolated from B. cenocepacia cultures grown in the LB standard medium without and with chloramphenicol. A) Lane $M$ represents the molecular weight marker (Fermentas), whose sizes of some bands in base pairs (bp) are provided on the left; lanes 3, 5, 7, 9, and II represent positive controls, i.e. genomic DNA amplification with primers specific for orf3, orf9, orfl 0, orfII, and orfl 3 (Table 3); lanes I and 2 represent RT-PCR amplification of orf3 in the absence and in the presence of $12.8 \mu \mathrm{g} / \mathrm{ml}(0.4 \mathrm{X} \mathrm{MIC})$ of chloramphenicol, respectively; lanes $4,6,8$, and I0 represent RT-PCR amplifications of orf9, orfl 0 , orfI I, and orfl3, without antibiotic, respectively; lane CT represents the PCR reaction negative control. B) Lanes I and 2 represent the expression of $r R N A$ I6S gene in the presence or in the absence of chloramphenicol, respectively; lane 3 represents the positive control, i.e. genomic DNA amplification with primers specific for rRNA I6S gene; lane $M$ represents the molecular weight marker (Fermentas), whose sizes of some bands in base pairs (bp) are provided on the right.

mation is available on the regulation of mexXY expression, except that mutations occurring in the divergently transcribed repressor gene mex $Z$ are frequently isolated from the sputa of cystic fibrosis patients [45]. In P. aeruginosa, with the exception of MexAB-OprM, the expression of most of the RND efflux systems is tightly regulated and overexpression of these RND pumps is usually caused by mutations in genes encoding regulatory proteins [39]. Notably, overexpression of multidrug resistance pumps in clinical isolates of $P$. aeruginosa, resulting in increased bacterial resistance, is usually due to mutations in these regulatory genes $[46,47]$. It would be interesting to further investigate if the expression of $\operatorname{orf} 3, \operatorname{orf} 9, \operatorname{orf11}$, and orf13 is due to a constitutive expression or to a transcriptional deregulation in the B. cenocepacia clinical isolate analysed in this study, which showed a multidrug resistance phenotype as it is resistant to ampicillin and aztreonam (>2048 $\mu \mathrm{g} / \mathrm{ml})$, ceftazidime $(768 \mu \mathrm{g} / \mathrm{ml})$, meropenem $(64 \mu \mathrm{g} /$ $\mathrm{ml})$, piperacillin $(768 \mu \mathrm{g} / \mathrm{ml})$ amikacin $(64 \mu \mathrm{g} / \mathrm{ml})$, nor- floxacin $(32 \mu \mathrm{g} / \mathrm{ml})$, rifampicin $(256 \mu \mathrm{g} / \mathrm{ml})$, streptomycin $(>2048 \mu \mathrm{g} / \mathrm{ml})$, tetracycline $(128 \mu \mathrm{g} / \mathrm{ml})$, chloramphenicol $(32 \mu \mathrm{g} / \mathrm{ml})$, and gentamicin $(1536 \mu \mathrm{g} /$ $\mathrm{ml})$.

\section{Cloning and phenotypic analysis of B. cenocepacia orf 2 into E. coli KAM3}

One approach to test the role of the identified genes in drug efflux is to clone them into suitable hosts by selecting on different compounds. In spite of several attempts, we met many problems in cloning experiments since a lot of rearranged clones were found in the host strains (E. coli and $B$. multivorans). The difficulties that we met in cloning experiments could rely both on plasmid instability and/or toxic effect of the gene product (data not shown). In fact, the failure could be due to the toxicity of the gene product for the host bacterial cells and still $B$. multivorans as a host causes many troubles (i.e. recombinations, overlapping with existing pumps, etc.). 
To overcome this problem we used the E. coli KAM3 strain as host, which lacks the $\operatorname{acr} A B$ genes and is sensitive to many drugs that are known as substrates of the AcrAB system [48]. We decided to characterize one of the 14 hypothetical drug efflux pumps of $B$. cenocepacia by cloning the orf2-coding region from $B$. cenocepacia genomic DNA into pBAD202 expression vector. The pBAD202 vector and the recombinant plasmid ( $\mathrm{pBAD} /$ orf2) were transformed into E. coli KAM3. We chose ORF2 because of its high homology to MexB, responsible for antibiotic resistance in many Pseudomonas clinical isolates [3]. Particularly, studies with mutants that overproduce or lack MexAB-OprM demonstrated that this efflux system extrudes quinolones, macrolides, tetracycline, chloramphenicol, novobiocin, and most $\beta$-lactams but not imipenem $[3,49]$.

The MICs of E. coli KAM3 strains harbouring pBAD202 and pBAD/orf2, respectively, were determined by streaking the cultures onto LB containing different concentrations of the following compounds: streptomycin, ethidium bromide, nalidixic acid, several quinolones (ciprofloxacin, ofloxacin, norfloxacin, and sparfloxacin), chloramphenicol, erythromycin, tetraphenylphosphonium, and tetracycline. Plates were incubated at $37^{\circ} \mathrm{C}$ for 3 days and the growth was visually evaluated. Compared to the control strain, the overexpression of orf 2 into E. coli KAM3 cells conferred resistance to streptomycin (16X MIC), tetraphenylphosphonium (8X MIC), ethidium bromide (4X MIC), nalidixic acid (4X MIC), ciprofloxacin (4X MIC), ofloxacin (2X MIC), and norfloxacin (2X MIC) (Table 2). No difference in drug susceptibility was found with sparfloxacin, chloramphenicol, erythromycin, and tetracycline (data not shown). These data suggest that orf2 is expressed and functional in E. coli KAM3. Interestingly, in $P$. aeruginosa, it has been found that fluoroquinolones like nalidixic acid and norfloxacin can commonly select mutants that constitutively overproduce the MexABOprM efflux pump system [46].

From these data it appeared that streptomycin and norfloxacin could be substrates of ORF2. We decided to perform RT-PCR of B. cenocepacia grown in the presence of sub-inhibitory concentration of streptomycin $(204.8 \mu \mathrm{g} /$ $\mathrm{ml})$ and norfloxacin $(3.2 \mu \mathrm{g} / \mathrm{ml})$. In our condition, orf2 transcript could not be detected by RT-PCR (data not shown). The expression of orf2 was also evaluated by Southern blotting of the RT-PCR agarose gel and subsequent hybridization with a labelled 511-bp fragment internal to orf2 coding region. No hybridisation signals were observed (data not shown), indicating that orf2 is not expressed following treatment of B. cenocepacia cells with streptomycin or norfloxacin.

Three different hypotheses could be given to explain the fact that orf 2 is not expressed. First, the experimental approaches utilized in this work (RT-PCR and Southern hybridization analysis) are not enough sensitive to detect very small amount of orf 2 mRNA. Real-time PCR is an attractive method for estimating gene expression of efflux pumps in bacteria because of its great sensitivity and wide effective range. Recently, the real-time PCR appeared to be a useful alternative method for assessing the expression of efflux pumps MexB and MexY in P. aeruginosa [50]. Second, not all ORF2 substrates in $E$. coli necessarily must be inducers of the expression of this pump in B. cenocepacia. A relevant example of such a behaviour is given by the MexCD-OprJ efflux pump of $P$. aeruginosa, which extrudes antibiotics that do not induce its expression [51]. Instead, expression of this efflux system was induced by clinical important disinfectants such as benzalkonium chloride and chlorhexidine gluconate [51]. Finally, the orf2 expression may occur in a phase of cellular growth different from the exponential one we tested. In conclusion, we could not determined neither the real inducing agent nor the culture conditions in which induction of orf 2 expression is triggered; therefore, it could be interesting to test other organic compounds for the ability to induce expression of this efflux pump.

\section{Ethidium bromide efflux activity}

Ethidium bromide efflux experiment was performed to determine whether E. coli KAM3 cells, carrying the recombinant plasmid pBAD/orf2, were more resistant to this dye due to an active efflux mechanism.

As shown in Figure 3, cells harboring the cloning vector pBAD202 take up ethidium bromide rapidly and achieve

Table 2: MIC $(\mu \mathrm{g} / \mathrm{ml})$ of drugs for E. coli KAM3 cells transformed with pBAD202 and pBAD/orf2 plasmids

\begin{tabular}{lll}
\hline & pBAD202 & pBAD/orf2 \\
\hline Streptomycin & 0.5 & $8(16 X)$ \\
Tetraphenylphosphonium & 4 & $32(8 X)$ \\
Ethidium bromide & 8 & $32(4 X)$ \\
Nalidixic acid & 0.25 & $1(4 X)$ \\
Norfloxacin & 0.0125 & $0.025(2 X)$ \\
Ofloxacin & 0.002 & $0.004(2 X)$ \\
Ciprofloxacin & 0.001 & $0.004(4 X)$ \\
\hline
\end{tabular}




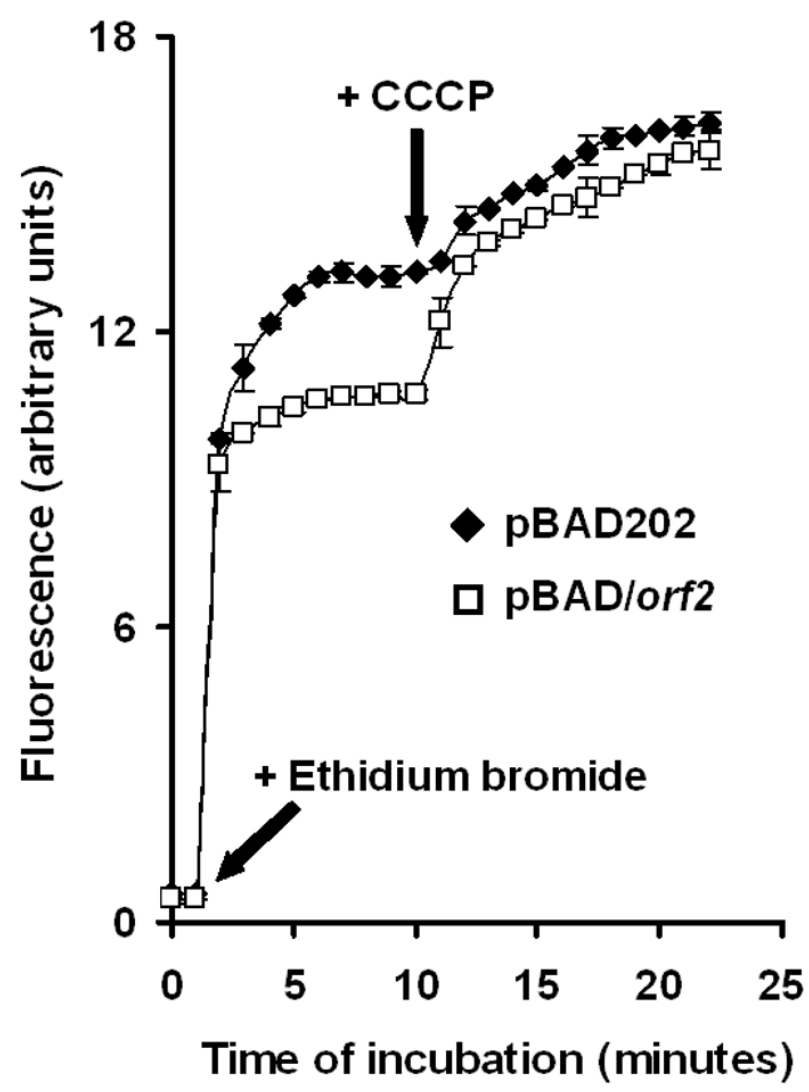

Figure 3

Accumulation of ethidium bromide in E. coli KAM3 cells harbouring pBAD202 and pBAD/orf2. E. coli KAM3 cells containing the cloning vector PBAD202 and the recombinant plasmid pBAD/orf2 were grown in LB medium at $37^{\circ} \mathrm{C}$ and treated as described in Methods. Ethidium bromide was added to cell suspensions carrying pBAD202 ( or pBAD/orf2 $(\square)$, at a final concentration of $20 \mu \mathrm{M}$ whereas the efflux inhibitor CCCP was added 9 min after the addition of ethidium bromide at a final concentration of $30 \mu \mathrm{M}$. The experimental error bars showed standard deviations of triplicate determinations.

a steady-state level of accumulation within about 5 min of incubation.

As observed in several experiments, accumulation of ethidium bromide was approximately 20\% lower when cells contained the pBAD/orf2 recombinant plasmid.

A reduced level of accumulation of the drug may be caused either by a decreased level of drug permeation or by active drug extrusion through the cytoplasmic membrane. To study the effect of membrane deenergization on the uptake of tetracycline, the protonophore carbonyl cya- nide $m$-chlophenylhydrazone (CCCP) was added to cells containing ethidium bromide. Upon the addition of CCCP, the level of ethidium bromide accumulation increased in the case of the pBAD/orf2-harboring strain and reached a level almost equal to that observed in the case of the strain containing only the cloning vector pBAD202 (Figure 3). On the contrary, under our conditions, CCCP had no significant effect on the level of ethidium bromide accumulation in the strain carrying the cloning vector (Figure 3). These data indicate that ORF2 pumped out ethidium bromide in an energy-dependent process, presumably by using proton motive force. We could conclude that ORF2, belonging to RND transporters, is an efflux pump of B. cenocepacia J2315.

\section{Conclusion}

Pulmonary infection with Bcc in patients with CF is often associated with a more rapid decline in lung function. Because of the resistance of Bcc to many antibiotics, treatment options are often limited.

The emergence of active efflux as a major causative factor in antibiotic resistance has been one of the most significant trend in anti-infective chemotherapy over the last decade and strategies to identify efflux pump inhibitors are in progress [52]. For these reasons, the identification and the characterization of such efflux systems in B. cenocepacia are a very important topic in the field of antibiotic resistance.

Taken together the results described in this paper fit with the prediction that $B$. cenocepacia contains homologs of the $P$. aeruginosa Mex-Opr efflux pump systems. Moreover, orf3, orf9, orf11, and orf13 are expressed in B. cenocepacia J2315, even if, at this stage, it is not possible to state if this expression is constitutive or due to mutations in regulatory genes.

Finally, the results described in this paper suggested that at least one of the RND-type efflux pumps identified, extrude out of the cell some antibiotics as well as other compounds (e.g. ethidium bromide). To demonstrate that this pump is really involved in the multidrug resistant phenotype of the B. cenocepacia J2315, further studies are necessary. The generation of mutants with pump overproduction and/or genetic deletion of genes encoding pumps in a controlled laboratory environment provides invaluable information on the potential impact of multidrug efflux pumps on the intrinsic resistance of $B$. cenocepacia J2315. Because of the difficulties in cloning and expression experiments, knock-out approach represents the only alternative method to physiologically characterize these transporters and to confirm if the encoded pumps play a significant role in the antimicrobial resistance of this microorganism. 
Table 3: Primers used in this study

\begin{tabular}{|c|c|c|c|}
\hline Primer & Sequence of primers & Comments & Product size (bp) \\
\hline 2pbadFOR & 5'-CACCATGGCCAAGTTCTTTATC-3' & Sense primer for the cloning of orf2-coding into pBAD202 & 3192 \\
\hline 2pbadREV & 5'-TTACTGCCCCTTCGAATCGTTT-3' & Antiense primer for the cloning of orf2-coding into pBAD 202 & \\
\hline IrtFOR & 5'-CCACGCCGTTCGTTCGCATCT-3' & Sense primer for RT-PCR analysis of orfI & 577 \\
\hline I rtREV & 5'-GCTTGGCGAACCACCGGTTGA-3' & Antisense primer for RT-PCR analysis orfl & \\
\hline 2rtFOR & 5'-CGAAGCTGAAGCCGTTGAC-3' & Sense primer for RT-PCR analysis orf2 & 511 \\
\hline 2rtREV & 5'-TCGTGCTGTCGGCGGTGTT-3' & Antisense primer for RT-PCR analysis orf2 & \\
\hline 3rtFOR & 5'-CGCGCTCACCGACCTGATGTT-3' & Sense primer for RT-PCR analysis orf3 & 531 \\
\hline 3rtREV & 5'-AGCGCGAGGAACACGACGAGC-3' & Antisense primer for RT-PCR analysis orf3 & \\
\hline 4rtFOR & 5'-AACATCCTGCGCTGAAGGTCAAC-3' & Sense primer for RT-PCR analysis orf 4 & 537 \\
\hline 4rtREV & 5'-TGACAGCCCCTCTTCCGCCATCAC-3' & Antisense primer for RT-PCR analysis orf4 & \\
\hline 5rtFOR & 5'-TACGTGCTGCGCGAACTGAAG-3' & Sense primer for RT-PCR analysis orf5 & 594 \\
\hline 5rtREV & 5'-AACAGCCACATCACGCAAAACAC-3' & Antisense primer for RT-PCR analysis orf5 & \\
\hline 6rtFOR & 5'-TCGAATGGGTGCTGAACCGC-3' & Sense primer for RT-PCR analysis orf6 & 477 \\
\hline 6rtREV & 5'-CGAATTCTTCCGAGTTGGGGCT-3' & Antisense primer for RT-PCR analysis orf6 & \\
\hline 8rtFOR & 5'-CAAGGTCGTGACCGGCGTGTA-3' & Sense primer for RT-PCR analysis orf8 & 517 \\
\hline 8rtREV & 5'-GATCATCGACAGCACCATCAGC-3' & Antisense primer for RT-PCR analysis orf8 & \\
\hline 9rtFOR & 5'-CTATTCGATCAATACGCTCACG-3' & Sense primer for RT-PCR analysis orf9 & 493 \\
\hline 9rtREV & 5'-CGCATAGACGGCGAACACCAC-3' & Antisense primer for RT-PCR analysis orf9 & \\
\hline IOrtFOR & 5'-AACTCGCTGCAGATCTCGGAC-3' & Sense primer for RT-PCR analysis orf 10 & 521 \\
\hline IOrtREV & 5'-ATCGCGAACTGCTTGTAGAACTG-3' & Antisense primer for RT-PCR analysis orf 10 & \\
\hline I IrtFOR & 5'-TCGCGGCGAAGATGGAGGAC-3' & Sense primer for RT-PCR analysis orf I I & 522 \\
\hline I IrtREV & 5'-TCGTCGCCATCGGATGGAACAT-3' & Antisense primer for RT-PCR analysis orf I I & \\
\hline I 2rtFOR & 5'-CGCGCTCAACGACTGGTTCCT-3' & Sense primer for RT-PCR analysis orf 12 & 520 \\
\hline I 2rtREV & 5'-CAGTTGCGATCGGTCGTAGGTC-3' & Antisense primer for RT-PCR analysis orf 12 & \\
\hline I3rtFOR & 5'-CAGGTTACACAGATGCGAACGA-3' & Sense primer for RT-PCR analysis orf 13 & 530 \\
\hline I3rtREV & 5'-AAGCCCGACACGTGACGAGCG-3' & Antisense primer for RT-PCR analysis orf 13 & \\
\hline I4rtFOR & 5'-ACGCCGACCGACATCTTTCCG-3' & Sense primer for RT-PCR analysis orf 14 & 615 \\
\hline I4rtREV & 5'-CTGCGTGCTGCCGTTCATCTGC-3' & Antisense primer for RT-PCR analysis orfl 4 & \\
\hline I5rtFOR & 5'-AGAGTTTGATCATGGCTCAG-3' & Sense primer for RT-PCR analysis $r R N A$ I6S gene & 1490 \\
\hline I6rtREV & 5'-TACCTTGTTACGACTTCAC-3' & Antisense primer for RT-PCR analysis rRNA I6S gene & \\
\hline
\end{tabular}


A greater understanding of the genetic determinants that play a role in antibiotic resistance will lead to the development of new strategies for the treatment of B. cenocepacia infections in patients with cystic fibrosis.

\section{Methods \\ Computer methods}

The B. cenocepacia J2315 genome sequence data were produced by the B. cenocepacia Sequencing Group at the Sanger Institute and it was retrieved from the Sanger FTP Server [53]. This analysis integrates different approaches and is based on the conservation of three features among drug transporters in microorganisms: specific sequence motifs, overall sequence similarity, and structural similarities.

Searches of the stretches of amino acids containing the specific motifs conserved in members of the RND transporters among the B. cenocepacia proteins were done using the BLAST SEARCH program provided at the Sanger website [33]. To well characterize the specific motifs present in the $B$. cenocepacia transporters, the regions containing the motifs were aligned using the CLUSTALW program within the EBI website [54]. Searches of sequence similarity between potential drug transporters and proteins characterized in $E$. coli and $P$. aeruginosa were done using the BLASTP program within the NCBI website [55]. The number and the position of the transmembrane domains (TMS) have been predicted by TMHMM program within the CBS website [56]. All the identified hypothetical RND proteins were analysed by InterProScan program provided by the EBI website [54] and Phyre program provided by the SBG website [57]. InterPro is a database of protein families, domains, and functional sites in which identifiable features found in known proteins can be applied to unknown protein sequences; the Phyre automatic fold recognition server predicts the structure and/or function of unknown protein sequences.

The analysis for the presence of duplex destabilized motifs in sequences containing the intergenic and coding regions encompassing RND genes were done using WEBSIDD program within the Genome Center website [58]. This program uses an algorithm that allows to identify regions of Stress-Induced DNA duplex Destabilization (SIDD) that could be associated with promoters [59].

\section{Growth conditions, plasmids, and cloning procedures}

Escherichia coli KAM3 and Burkholderia cenocepacia J2315 strains were grown on Luria Bertani agar or broth (LB) medium at $37^{\circ} \mathrm{C}$. E. coli KAM3 cells harbouring pBAD202 and $\mathrm{pBAD} /$ orf 2 plasmids were grown in the presence of kanamycin $(50 \mu \mathrm{g} / \mathrm{ml})$ (Sigma).
B. cenocepacia $\mathrm{J} 2315$ genomic DNA was isolated as described previously by Scordilis et al. [60].orf2 was amplified by PCR from B. cenocepacia J2315 genomic DNA, by using the 2pbadFOR and 2pbadREV primers indicated in Table 3 . The CACC sequence was introduced at 5 terminus of the primer 2 pbadFOR to directionally clone the PCR product in the correct orientation into pBAD202 expression vector (as described by "pBAD directional TOPO expression kit" of Invitrogen).

The PCR reaction was carried out as follows by using AccuPrime Pfx DNA polymerase (Invitrogen): initial denaturation at $95^{\circ} \mathrm{C}$ for $3 \mathrm{~min} ; 35$ cycles of denaturation at $95^{\circ} \mathrm{C}$ for $30 \mathrm{sec}$, annealing at $52^{\circ} \mathrm{C}$ for $1 \mathrm{~min}$, extension at $68^{\circ} \mathrm{C}$ for $4 \mathrm{~min}$ and final extension at $68^{\circ} \mathrm{C}$ for $10 \mathrm{~min}$. PCR products were analysed by electrophoresis on $1 \%$ agarose gel, containing $0.5 \mu \mathrm{g} / \mathrm{ml}$ of ethidium bromide and visualized under UV light.

The orf2 PCR product was cloned into pBAD202 expression vector and transformed into E. coli Top 10 competent cells (Invitrogen). Plasmid DNA, isolated by the alkaline lysis method [61], was sequenced to be sure that no mutations were introduced in the amplified product and introduced into E. coli KAM3 strain by electroporation.

\section{RT-PCR}

RT-PCR was used to monitor gene expression. B. cenocepacia J2315 strain was grown to O.D. ${ }_{600}=0.3$ in LB broth. To detect if gene expression was induced by some substrates, cells were grown in the presence of chloramphenicol at a final concentration of $12.8 \mu \mathrm{g} / \mathrm{ml}(0.4 \mathrm{X}$ MIC), streptomycin at a final concentration of $204.8 \mu \mathrm{g} / \mathrm{ml}$ (0.1X MIC), and norfloxacin at a final concentration of $3.2 \mu \mathrm{g} / \mathrm{ml}$ (0.1X MIC). Total RNA was extracted using the RNeasy Mini Kit (Qiagen), according to the manufacturer's protocol. $1 \mu \mathrm{g}$ of RNA was treated with $10 \mathrm{U}$ of DNase I-RNase free (Roche) for $30 \mathrm{~min}$ at room temperature and then heated at $70^{\circ} \mathrm{C}$ for $10 \mathrm{~min}$. The RT reactions were carried out, by using $2 \mu \mathrm{g}$ of template RNA in the presence of $\mathrm{M}$ MLV Reverse Transcriptase (Promega) and specific gene primers (Table 3 ). Reverse transcription was carried out as follows: (a) $2 \mu \mathrm{g}$ of RNA and $0.5 \mu \mathrm{g}$ of each downstream primer were incubated at $70^{\circ} \mathrm{C}$ for $5 \mathrm{~min}$ and then cooled on ice; (b) $5 \mu \mathrm{l}$ of $5 \mathrm{X}$ reaction buffer, $5 \mu \mathrm{l}$ of $10 \mathrm{mM}$ dNTPs, and $200 \mathrm{U}$ of M-MLV enzyme were added and the reaction was carried out at $37^{\circ} \mathrm{C}$ for $1 \mathrm{~h}$; (c) the enzyme was inactivated at $95^{\circ} \mathrm{C}$ for $5 \mathrm{~min}$ and the reaction was ethanol-precipitated. The cDNA was dissolved in $20 \mu \mathrm{l}$ of deionised water and $4 \mu \mathrm{l}$ were used as template with the gene-specific primers (Table 3 ) for PCR amplification, by using RedTaq genomic DNA polymerase (Sigma). The PCR reaction was carried out as follows: initial denaturation at $94^{\circ} \mathrm{C}$ for $3 \mathrm{~min} ; 25$ cycles of denaturation at $94^{\circ} \mathrm{C}$ for $1 \mathrm{~min}$, annealing at $54^{\circ} \mathrm{C}, 62^{\circ} \mathrm{C}, 64^{\circ} \mathrm{C}, 66^{\circ} \mathrm{C}$ or $68^{\circ} \mathrm{C}$ 
depending on the primer for $1 \mathrm{~min}$, extension at $72^{\circ} \mathrm{C}$ for $1 \mathrm{~min}$, and final extension at $72^{\circ} \mathrm{C}$ for $10 \mathrm{~min}$. RT-PCR products were analysed by electrophoresis on $1.5 \%$ agarose gel, containing $0.5 \mu \mathrm{g} / \mathrm{ml}$ of ethidium bromide and visualized under UV light. The same reactions were carried out for each sample without M-MLV Reverse Transcriptase to ensure that amplification was a result of cDNA and not of contaminating DNA molecules. In all experiments, the expression of rRNA $16 \mathrm{~S}$ gene was determined as an internal control to ensure that the differences observed in genes expression were not due to variability in the RNA isolation and/or in the RT-PCR technique.

Southern blotting was performed as described by Sambrook and Russell [61]; a 511-bp fragment internal to orf2 coding region was labelled with $\left[\alpha-{ }^{32} \mathrm{P}\right]$ dCTP $3000 \mathrm{Ci}$ $\mathrm{mmol}^{-1}$ (Amersham Biosciences) by using the HexaLabel Plus DNA Labelling Kit (Fermentas) according to the manufacturer's instructions.

\section{MIC determinations}

Determination of MIC for E. coli KAM3, transformed with recombinant plasmids, was performed by streaking $1 \times$ $10^{5}$ cells onto LB agar containing different concentrations of drugs. The following compounds were tested: streptomycin, ethidium bromide, nalidixic acid, several quinolones (ciprofloxacin, ofloxacin, norfloxacin, and sparfloxacin), chloramphenicol, tetraphenylphosphonium erythromycin, and tetracycline. Plates were incubated at $37^{\circ} \mathrm{C}$ for 3 days and the growth was visually evaluated.

Determination of MIC for B. cenocepacia J2315 strain was performed by streaking $1 \times 10^{5}$ cells onto LB containing different concentrations of drugs. Plates were incubated at $37^{\circ} \mathrm{C}$ for about 3 days and the growth was visually evaluated. The following antibiotics were tested to determine the resistance profile: ampicillin, amikacin, aztreonam, ceftazidime, chloramphenicol, gentamicin, meropenem, norfloxacin, piperacillin, tetracycline, streptomycin, and rifampicin.

The MIC is defined as the lowest concentration of drug that prevented visible growth. The results are the averages of three replicates.

\section{Ethidium bromide accumulation assay}

E. coli KAM3 cells harbouring pBAD202 and pBAD/orf2 plasmids were grown in LB medium in the presence of kanamycin $(50 \mu \mathrm{g} / \mathrm{ml})$ under aerobic conditions at $37^{\circ} \mathrm{C}$. Bacteria were harvested at the esponential phase of growth, washed twice with a minimal medium $[76 \mathrm{mM}$ $\left(\mathrm{NH}_{4}\right)_{2} \mathrm{SO}_{4}, 500 \mathrm{mM} \mathrm{KH} \mathrm{PO}_{4}, \mathrm{pH} 7.0,1 \mathrm{mM} \mathrm{MgSO}_{4}$, $0.4 \%$ glucose], and resuspended in the same medium to an O.D. ${ }_{650}$ of 0.2. Ethidium bromide was added to cells to a final concentration of $20 \mu \mathrm{M}$ and the change in fluorescence intensity was continuously monitored at excitation and emission wavelengths of 500 and $580 \mathrm{~nm}$, respectively. Fluorescence intensity is proportional to the quantity of intracellular dye, since ethidium bromide fluorescence is enhanced by the binding to intracellular components, especially to nucleic acids.

The efflux pumps inhibitor carbonyl cyanide $m$-chlophenylhydrazone (CCCP) was added to the cells suspensions to a final concentration of $30 \mu \mathrm{M}$ after 9 min of the addition of ethidium bromide, to assess energy-dependent efflux.

\section{Authors' contributions}

PG: carried out BLAST SEARCH, BLASTP, CLUSTALW, and TMHMM analyses, and efflux experiments. MRP: performed cloning experiments, MIC determinations, and manuscript preparation. EDR: performed experimental coordination. SB: performed RT-PCR experiments. PA: performed analysis of RND proteins by InterProScan and Phyre programs, and RND genes analysis by WEBSIDD program. GM: several cloning attempts with different vectors. GR: design of the study and coordination. All authors read and approved the final manuscript.

\section{Acknowledgements}

This research was supported by a grant from Italian Cystic Fibrosis Research Foundation (FFC). The project was adopted by FFC Delegation of Trento and by Zini and Furlini families. We thank M. Valvano for supplying E. coli KAM3 strain and G. Manno for supplying $B$. cenocepacia 2315 strain. The $B$. cenocepacia J23 15 genome sequence data were produced by the $B$. cenocepacia Sequencing Group at the Sanger Institute.

P.G. was supported by a fellowship from the FFC, Verona, Italy.

\section{References}

I. Nikaido H: Preventing drug access to targets: cell surface permeability barriers and active efflux in bacteria. Semin Cell Dev Biol 2001, I 2:21 5-223.

2. Ryan BM, Dougherty T], Beaulieu D, Chuang J, Dougherty BA, Barrett JF: Efflux in bacteria: what do we really know about it? Expert Opin Investig Drugs 2001, I 0: I 409-1422.

3. Li XZ, Nikaido $\mathrm{H}$, Poole K: Role of mexA-mexB-oprM in antibiotic efflux in Pseudomonas aeruginosa. Antimicrob Agents Chemother 1995, 39:1948-1953.

4. $\mathrm{Li} X Z$, Nikaido $\mathrm{H}$ : Efflux-mediated drug resistance in bacteria. Drugs 2004, 64: I59-204.

5. Paulsen IT, Brown MH, Skurray RA: Proton-dependent multidrug efflux systems. Microbiol Rev 1996, 60:575-608.

6. Akama H, Matsuura T, Kashiwagi S, Yoneyama H, Narita S, Tsukihara T, Nakagawa A, Nakae T: Crystal structure of the membrane fusion protein, MexA, of the multidrug transporter in Pseudomonas aeruginosa. J Biol Chem 2004, 279:25939-25942.

7. Akama $\mathrm{H}$, Kanemaki $M$, Yoshimura $M$, Tsukihara $T$, Kashiwagi $T$, Yoneyama H, Narita S, Nakagawa A, Nakae T: Crystal structure of the drug discharge outer membrane protein, OprM, of Pseudomonas aeruginosa: dual modes of membrane anchoring and occluded cavity end. J Biol Chem 2004, 279:52816-528I9.

8. Higgins MK, Bokma E, Koronakis E, Hughes C, Koronakis V: Structure of the periplasmic component of a bacterial drug efflux pump. Proc Natl Acad Sci USA 2004, I 0 I:9994-9999. 
9. Koronakis V, Sharff A, Koronakis E, Luisi B, Hughes C: Crysta structure of the bacterial membrane protein TolC central to multidrug efflux and protein export. Nature 2000, 405:9|4-919.

10. Murakami S, Nakashima R, Yamashita E, Yamaguchi A: Crystal struc ture of bacterial multidrug efflux transporter AcrB. Nature 2002, 419:587-593.

II. Koronakis V, Eswaran J, Hughes C: Structure and function of TolC: the bacterial exit duct for proteins and drugs. Annu Rev Biochem 2004, 73:467-489.

12. Aires JR, Nikaido $\mathrm{H}$ : Aminoglycosides are captured from both periplasm and cytoplasm by the AcrD multidrug efflux transporter of Escherichia coli. J Bacteriol 2005, I 87:1923-1929.

13. Eswaran J, Koronakis E, Higgins MK, Hughes C, Koronakis V: Three's company: component structures bring a closer view of tripartite drug efflux pumps. Curr Opin Struct Biol 2004, I 4:74I-747.

14. Ma D, Cook DN, Alberti M, Pon NG, Nikaido HM, Hearst JE: Molecular cloning of acrA and acrE genes of Escherichia coli. J Bacteriol 1993, I75:6299-63।3.

15. Aires JR, Kohler T, Nikaido H, Plesiat P: Involvement of an active efflux system in the natural resistance of Pseudomonas aeruginosa to aminoglycosides. Antimicrob Agents Chemother 1999 , 43:2624-2628

16. Poole K: Multidrug efflux pumps and antimicrobial resistance in Pseudomonas aeruginosa and related organisms. J Mol Microbiol Biotechnol 2001, 3:255-264.

17. Speert DP: Understanding Burkholderia cepacia: epidemiology, genomovars, and virulence. Infect Med 200I, I 8:49-56

18. Lyczak JB, Cannon CL, Pier GB: Lung infections associated with cystic fibrosis. Clin Microbiol Rev 2002, I 5:194-222.

19. Coenye T, Vandamme P, Govan JRW, LiPuma JJ: Taxonomy and identification of the Burkholderia cepacia complex. J Clin Microbiol 200I, 39:3427-3436.

20. Mahenthiralingam E, Urban TA, Goldberg JB: The multifarious, multireplicon Burkholderia cepacia complex. Nat Rev Microbiol 2005, 3: I44-I56.

21. Reik R, Spilker T, LiPuma J]: Distribution of Burkholderia cepacia complex species among isolates recovered from persons with or without cystic fibrosis. J Clin Microbiol 2005 , 43:2926-2928.

22. Quinn JP: Clinical problems posed by multiresistant nonfermenting gram-negative pathogens. Clin Infect Dis 1998, I:SI 17-124

23. Aronoff SC: Outer membrane permeability in Pseudomonas cepacia: diminished porin content in a beta-lactam-resistant mutant and in resistant cystic fibrosis isolates. Antimicrob Agents Chemother 1988, 32:1636-1639.

24. Moore RA, Hancock RE: Involvement of outer membrane of Pseudomonas cepacia in aminoglycoside and polymyxin resistance. Antimicrob Agents Chemother 1986, 30:923-926.

25. Parr TR Jr, Moore RA, Moore LV, Hancock RE: Role of porins in intrinsic antibiotic resistance of Pseudomonas cepacia. Antimicrob Agents Chemother 1987, 3 I: I 2 I- I 23.

26. Trepanier S, Prince A, Huletsky A: Characterization of the penA and pen $R$ genes of Burkholderia cepacia 249 which encode the chromosomal class A penicillinase and its LysR-type transcriptional regulator. Antimicrob Agents Chemother 1997, 4I:2399-2405

27. Burns JL, Lien DM, Hedin LA: Isolation and characterization of dihydrofolate reductase from trimethoprim-susceptible and trimethoprim-resistant Pseudomonas cepacia. Antimicrob Agents Chemother 1989, 33:I247-125I.

28. Fehlner-Gardiner CC, Valvano MA: Cloning and characterization of the Burkholderia vietnamiensis norM gene encoding a multidrug efflux protein. FEMS Microbiol Lett 2002, 2 I 5:279-283.

29. Wigfield SM, Rigg GP, Kavari M, Webb AK, Matthews RC, Burnie JP: Identification of an immunodominant drug efflux pump in Burkholderia cepacia. I Antimicrob Chemother 2002, 49:619-624.

30. Burns JL, Wadsworth CD, Barry J], Goodall CP: Nucleotide sequence analysis of a gene from Burkholderia (Pseudomonas) cepacia encoding an outer membrane lipoprotein involved in multiple antibiotic resistance. Antimicrob Agents Chemother 1996, 40:307-313

31. Nair BM, Cheung KJ Jr, Griffith A, Burns JL: Salicylate induces an antibiotic efflux pump in Burkholderia cepacia complex genomovar III (B. cenocepacia). J Clin Invest 2004, I I 3:464-473.
32. Govan JR, Brown PH, Maddison J, Doherty CJ, Nelson JW, Dodd M, Greening AP, Webb AK: Evidence for transmission of Pseudomonas cepacia by social contact in cystic fibrosis. Lancet 1993, 342:15-19.

33. The Sanger Centre [http://www.sanger.ac.uk]

34. Putman M, van Veen HW, Konings WN: Molecular properties of bacterial multidrug transporters. Microbiol Mol Biol Rev 2000, 64:672-693.

35. Ma D, Cook DN, Alberti M, Pon NG, Nikaido H, Hearst JE: Genes acrA and $a c r B$ encode a stress-induced efflux system of Escherichia coli. Mol Microbiol 1995, 16:45-55.

36. Hassan MT, van der Lelie D, Springael D, Romling U, Ahmed N, Mergeay $M$ : Identification of a gene cluster, czr, involved in cadmium and zinc resistance in Pseudomonas aeruginosa. Gene 1999, 238:417-425.

37. Zgurskaya $\mathrm{HI}$, Nikaido $\mathrm{H}$ : Multidrug resistance mechanisms: drug efflux across two membranes. Mol Microbiol 2000 37:219-225

38. Nakamura $\mathrm{H}$ : Novel acriflavin resistance genes, acrC and acrD, in Escherichia coli K-I 2. J Bacteriol | 979, I 39:8-I2.

39. Schweizer HP: Efflux as a mechanism of resistance to antimicrobials in Pseudomonas aeruginosa and related bacteria: unanswered questions. Genet Mol Res 2003, 3 I:48-62.

40. Kumar A, Schweizer HP: Bacterial resistance to antibiotics: active efflux and reduced uptake. Adv Drug Deliv Rev 2005 , 57:|486-|5|3.

4I. Adewoye L, Sutherland A, Srikumar R, Poole K: The MexR repressor of the mexAB-oprM multidrug efflux operon in Pseudomonas aeruginosa: characterization of mutations compromising activity. J Bacteriol 2002, I 84:4308-43 I 2.

42. Wang $H$, Noordewier M, Benham Cl: Stress-induced DNA duplex destabilization (SIDD) in the $E$. coli genome: SIDD sites are closely associated with promoters. Genome Res 2004 | 4: | 575- | 584 .

43. Masuda N, Sakagawa E, Ohya S, Gotoh N, Tsujimoto H, Nishino T: Contribution of the MexX-MexY-OprM efflux system to intrinsic resistance in Pseudomonas aeruginosa. Antimicrob Agents Chemother 2000, 44:2242-2246.

44. Jeannot K, Sobel ML, El Garch F, Poole K, Plesiat P: Induction of the MexXY efflux pump in Pseudomonas aeruginosa is dependent on drug-ribosome interaction. J Bacteriol 2005, I 87:534I-5346.

45. Vogne C, Aires JR, Bailly C, Hocquet D, Plésiat P: Role of the multidrug efflux system MexXY in the emergence of moderate resistance to aminoglycosides among Pseudomonas aeruginosa isolates from patients with cystic fibrosis. Antimicrob Agents Chemother 2004, 48: I676-1680.

46. Aeschlimann JR: The role of multidrug efflux pumps in the antibiotic resistance of Pseudomonas aeruginosa and other gramnegative bacteria. Insights from the Society of Infectious Diseases Pharmacists. Pharmacotherapy 2003, 23:916-924.

47. Piddock LJV: Clinically relevant chromosomally encoded multidrug resistance efflux pumps in bacteria. Clin Microbiol Rev 2006, 19:382-402.

48. Morita Y, Kodama K, Shiota S, Mine T, Kataoka A, Mizushima T, Tsuchiya T: NorM, a putative multidrug efflux protein, of Vibrio parahaemolyticus and its homolog in Escherichia coli. Antimicrob Agents Chemother 1998, 42:1778-I782.

49. Masuda N, Ohya S: Cross-resistance to meropenem, cephems, and quinolones in Pseudomonas aeruginosa. Antimicrob Agents Chemother 1992, 36: I847-185।.

50. Yoneda K, Chikumi H, Murata T, Gotoh N, Yamamoto H, Fujiwara H, Nishino T, Shimizu E: Measurement of Pseudomonas aeruginosa multidrug efflux pumps by quantitative real-time polymerase chain reaction. FEMS Microbial Lett 2005, 243:|25-|3|.

5I. Morita Y, Murata T, Mima T, Shiota S, Kuroda T, Mizushima T, Gotoh $\mathrm{N}$, Nishino $\mathrm{T}$, Tsuchiya $\mathrm{T}$ : Induction of mexCD-opr] operon for a multidrug efflux pump by disinfectants in wild-type Pseudomonas aeruginosa PAOI. J Antimicrob Chemother 2003, 5 I:991-994.

52. Lomovskaya O, Watkins W]: Efflux pumps: their role in antibacterial drug discovery. Curr Med Chem 200I, 8:1699-I7II.

53. The Sanger FTP Server [ftp://ftp.sanger.ac.uk/pub/pathogens/bc/]

54. The European Bioinformatics Institute [http://www.ebi.ac.uk/]

55. National Centre for Biotechnology Information [http://] www.ncbi.nlm.nih.gov/] 
56. Center for Biological Sequence Analysis [http://
[ www.cbs.dtu.dk/]

57. Structural Bioinformatics Group [http://www.sbg.bio.ic.ac.uk/]

58. Genome Center [http://www.genomecenter.ucdavis.edu/]

59. Benham CJ: Sites of predicted stress-induced DNA duplex destabilization occur preferentially at regulatory loci. Proc Natl Acad Sci USA 1993, 90:2999-3003.

60. Scordilis GE, Ree H, Lessie TG: Identification of transposable elements which activate gene expression in Pseudomonas cepacia. J Bacteriol 1987, 169:8-13.

6I. Sambrook J, Russell DW: Molecular cloning: a laboratory manual. Cold Spring Harbor Laboratory Press. Cold Spring Harbor, NY; 2001.

Publish with Biomed Central and every scientist can read your work free of charge

"BioMed Central will be the most significant development for disseminating the results of biomedical research in our lifetime. "

Sir Paul Nurse, Cancer Research UK

Your research papers will be:

- available free of charge to the entire biomedical community

- peer reviewed and published immediately upon acceptance

- cited in PubMed and archived on PubMed Central

- yours - you keep the copyright

Submit your manuscript here:

http://www.biomedcentral.com/info/publishing_adv.asp
BioMedcentral 